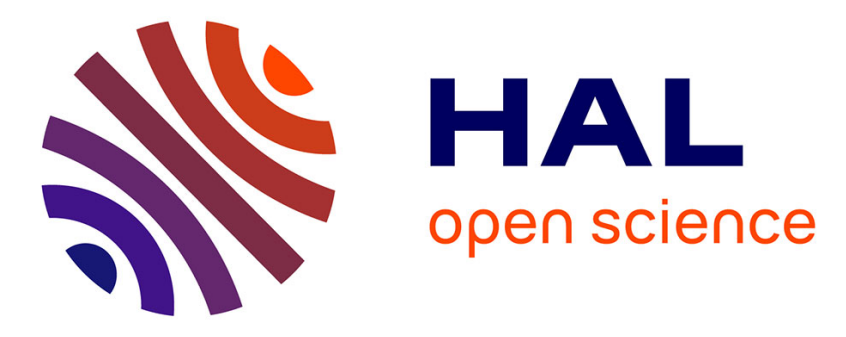

\title{
Incidence des cervicalgies en milieu professionnel Audrey Petit
}

\section{To cite this version:}

Audrey Petit. Incidence des cervicalgies en milieu professionnel. 28ème congrès français de rhumatologie, Dec 2015, Paris, France. 2015. hal-02615620

\section{HAL Id: hal-02615620 \\ https://univ-angers.hal.science/hal-02615620}

Submitted on 23 May 2020

HAL is a multi-disciplinary open access archive for the deposit and dissemination of scientific research documents, whether they are published or not. The documents may come from teaching and research institutions in France or abroad, or from public or private research centers.
L'archive ouverte pluridisciplinaire HAL, est destinée au dépôt et à la diffusion de documents scientifiques de niveau recherche, publiés ou non, émanant des établissements d'enseignement et de recherche français ou étrangers, des laboratoires publics ou privés. 


\section{Incidence des cervicalgies en milieu professionnel}

\author{
$\mathbf{L} \mathbf{U} \equiv$ \\ nam angers \\ PÔLE DE RECHERCHE ET D'ENSEIGNEMENT SUPÉRIEU
}

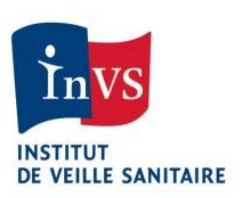

CHU ANGERS CENTRE HoSPTTLLIER
UNIVER STTAIRE

\section{Audrey Petit}

Laboratoire d'ergonomie et d'épidémiologie en santé au travail (LEEST), LUNAM Université

Centre de consultations de pathologie professionnelle du CHU d'Angers, France Institut de veille sanitaire (InVS), Département Santé-Travail, Saint-Maurice, France 


\section{Généralités sur les cervicalgies (1)}

\section{ÉPIDÉMIOLOGIE}

- La plupart des personnes ont fait ou feront l'expérience de la cervicalgie au cours de leur vie.

- Pour la majorité d'entre elles, la cervicalgie n'interfèrera pas de manière importante avec les activités habituelles.

- En population générale :

- prévalence entre 12,1 et $71,5 \%$ au cours des 12 derniers mois

- 1,7 à 11,5\% des cervicalgies entrainent une incapacité fonctionnelle

- Chez les travailleurs :

- prévalence entre 27,1 et $47,8 \%$ au cours des 12 derniers mois

- 11 à 14,1\% des cervicalgies entrainent une limitation des activités

- TOUS SECTEURS D'ACTIVITÉ 


\section{Généralités sur les cervicalgies (2)}

\section{FACTEURS DE RISQUE (FDR)}

- Etiologie multifactorielle +++

- FDR non modifiables : âge, genre, génétique

- FDR modifiables : tabagisme actif ou passif

- Facteur protecteur : activité physique sportive

- FDR professionnels reconnus :

- forte demande psychologique au travail

- faible soutien social

- posture statique prolongée

- travail répétitif

- travail de précision 


\section{Objectifs et méthodes de l'étude}

Objectifs :

- Évaluer l'incidence des cervicalgies en milieu professionnel

- Identifier les expositions professionnelles qui y sont associées

\section{Méthode :}

- Réseau pilote de surveillance des troubles musculo-squelettiques dans la population des salariés des Pays de la Loire, Institut de veille sanitaire (InVS)

- 1ère étape : constitution d'une cohorte de 3710 salariés des entreprises privées et publiques de la région des Pays de la Loire, par tirage au sort (2002-2005)

- $\underline{2^{e ̀ m e}}$ étape : envoi d'un auto-questionnaire postal (2007):

- taux de cervicalgies incidentes : cervicalgie $>8$ jours au cours des 12 derniers mois en l'absence de cervicalgie à l'inclusion

- conditions de travail et facteurs psychosociaux

- Analyses statistiques:

- associations entre cervicalgies incidentes et FDR personnels et professionnels

- pour chaque sexe séparément

- modèles de régressions logistiques multivariés 
non contactables : $\mathrm{n}=233$

non répondants : n=1 145

Sujets suivis en 2007-2009

$n=2332$

cervicalgies à l'inclusion $n=614$

données manquantes

lors du suivi : $n=29$

cervicalgies incidentes

$n=208$

Inactifs exclus : $\mathrm{n}=179$
Parmi les 2332 sujets de la cohorte, 1510 actifs ont été suivis :

- 10,4\% des hommes [8,4-12,4] déclaraient avoir souffert de cervicalgies pendant au moins 8 jours au cours des 12 derniers mois

- 14,6\% des femmes [11,8-17,4] déclaraient avoir souffert de cervicalgies pendant au moins 8 jours au cours des 12 derniers mois 


\section{Variables d'intérêt}

- Facteurs individuels : âge, indice de masse corporel, taille

- Facteurs organisationnels:

- durée de travail / variabilité de la durée de travail

- possibilité de quitter son travail des yeux / de pauses

- travail avec des collègues en situation précaire (CDD, intérimaires)

- rythme de travail imposé

- Facteurs biomécaniques:

- station assise prolongée

- position penchée en avant / penchée sur le côté

- port d'objets encombrants / pousser ou tirer des charges lourdes

- conduite prolongée d'engin de chantier / de véhicule

- travail au froid / utilisation d'outil vibrant

- travailler sur écran / sur clavier informatique

- travail tête penchée en avant / en arrière

- travail bras en l'air / éloignés du corps / accoudé

- pénibilité perçue du poste de travail (échelle de Borg)

- Facteurs psychosociaux:

- demande psychologique

- latitude décisionnelle (autonomie décisionnelle / utilisation des compétences )

- soutien social de la hiérarchie / des collègues. 


\section{Caractéristiques de la population}

- 3710 travailleurs inclus :

- 2161 hommes (58\%) et 1459 Femmes (42\%)

- âge moyen $=38,7 \pm 10,3$ ans

- $38 \%$ avaient une ancienneté dans le poste $>10$ ans

- échantillon représentatif de la population active de la région :

- industrie $=59 \%$

- agro-alimentaire $=34 \%$

- construction $=6 \%$

- agriculture $=1,5 \%$ 


\section{FDR de cervicalgies chez les hommes}

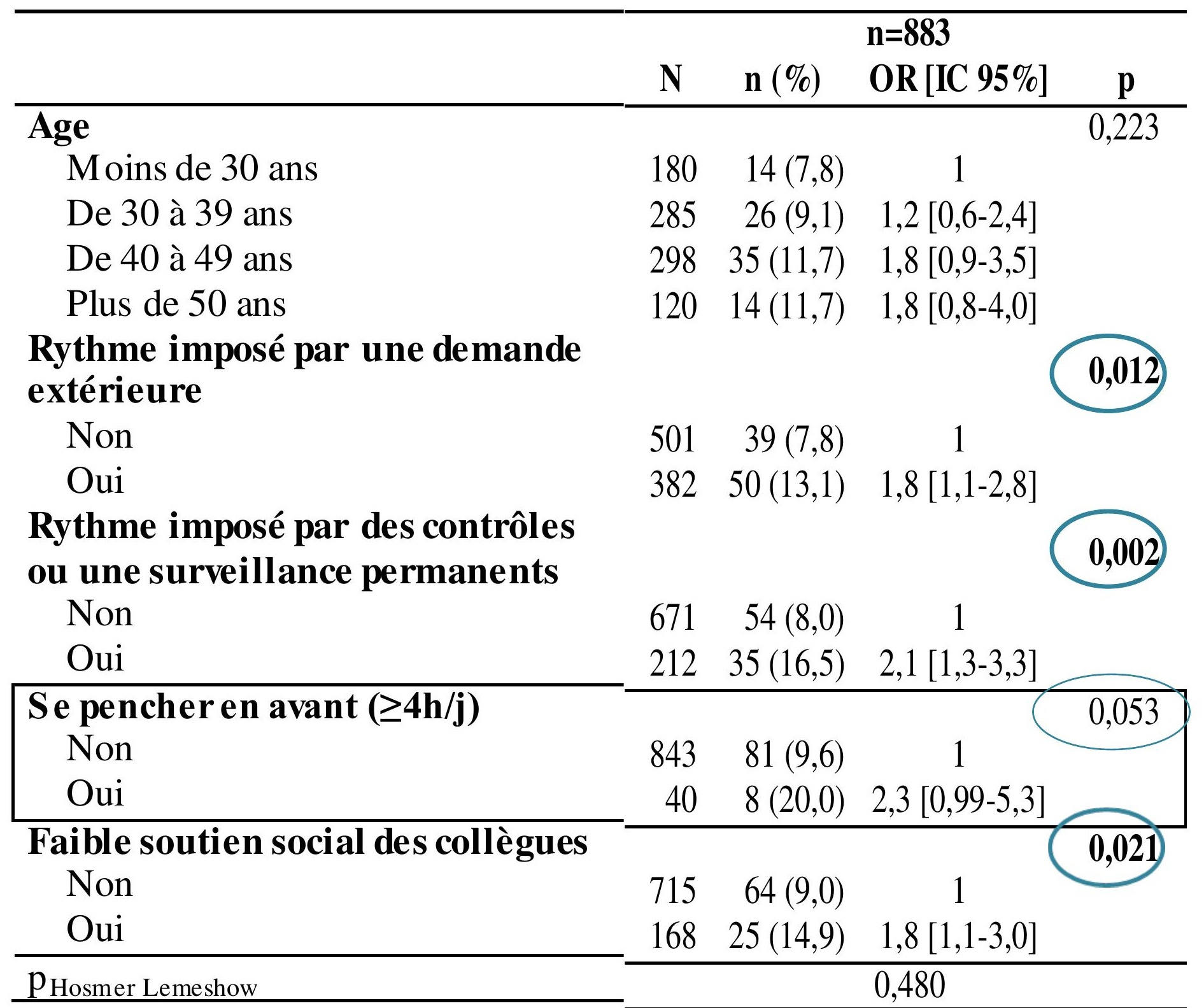




\section{FDR de cervicalgies chez les femmes}

\begin{tabular}{|c|c|c|c|c|}
\hline & \multicolumn{4}{|c|}{$\mathrm{n}=569$} \\
\hline & $\mathbf{n}$ & $\%$ & OR (IC 95\%) & p \\
\hline Age & & & & \\
\hline Moins de 30 ans & 105 & $13(12,4)$ & 1 & \\
\hline De 30 à 39 ans & 188 & $23(12,2)$ & $1,2[0,5-2,5]$ & \\
\hline De 40 à 49 ans & 199 & $39(19,6)$ & $2,3[1,1-4,6]$ & \\
\hline Plus de 50 ans & 77 & $9(11,7)$ & $1,2[0,5-3,1]$ & \\
\hline $\begin{array}{l}\text { Rythme imposé par le déplacement } \\
\text { d'un produit ou d'une pièce }\end{array}$ & & & & \\
\hline Non & 523 & $73(14,0)$ & 1 & \\
\hline Oui & 46 & $11(23,9)$ & $2,1[0,97-4,6]$ & \\
\hline $\begin{array}{l}\text { Travailler les bras en l'air ou les bras } \\
\text { éloignés du corps }(\geq 2 \mathrm{~h} / \mathbf{j}) \text { et Intensité } \\
\text { physique éle vée des efforts physiques } \\
(* \geq 14 \text { sur l'échelle } \mathrm{RPE} \text { de Borg) }\end{array}$ & & & & \\
\hline Aucun des deux (réf) & 390 & $48(12,3)$ & 1 & \\
\hline Adduction des bras seulement & 72 & $12(16,7)$ & $1,4[0,7-2,9]$ & \\
\hline Intensité phy sique élevée seulement & 64 & $10(15,6)$ & $1,4[0,6-2,9]$ & \\
\hline Les deux & 43 & $14(32,6)$ & $3,5[1,7-7,2]$ & \\
\hline
\end{tabular}




\section{Discussion - Conclusion}

- Large population de travailleurs salariés = représentativité

- Relativement peu de données dans la littérature sur l'incidence des cervicalgies dans des populations de travailleurs exposées à des niveaux de contraintes variés

- L'étude confirme :

- la forte incidence des cervicalgies en milieu professionnel

- la prédominance féminine

- le caractère multifactoriel de l'incidence des cervicalgies

- FDR principalement d'ordres psychosociaux et organisationnels pour les deux sexes

- Influence de l'âge et de la charge biomécanique de la ceinture scapulaire au travail chez les femmes 